\title{
Staphylococcus aureus meningitis from osteomyelitis of the spine
}

\author{
H.S. Markus and S.P. Allison \\ Department of Medicine, University Hospital, Queen's Medical Centre, Nottingham NG7 2UH, UK.
}

\begin{abstract}
Summary: Two cases of vertebral osteomyelitis presenting with secondary Staphylococcus aureus meningitis are described. In staphylococcal meningitis a search for a primary source should include the lower vertebral spine.
\end{abstract}

\section{Introduction}

Staphyloccus aureus is an uncommon cause of meningitis accounting for $1-9 \%$ of all bacterial causes. ${ }^{1}$ It is characterized by a high mortality and is frequently indicative of an underlying focus of infection. There are few reports of its association with vertebral osteomyelitis; we report two such cases.

\section{Case reports}

\section{Case 1}

A 60 year old chronic schizophrenic in long term institutional care was admitted with a few days history of increasing confusion. He had lost $7 \mathrm{lb}$ in weight over the last few weeks. He had recently finished a course of amoxycillin for a chest infection. On admission he was pyrexial and mildly confused. Investigations revealed a haemoglobin of $9.9 \mathrm{~g} / \mathrm{dl}$ and an ESR of $114 \mathrm{~mm} / \mathrm{h}$. The following day he became drowsy and developed neck stiffness. Brain computed tomographic (CT) scan was normal. Lumbar puncture showed normal pressure, protein $0.7 \mathrm{~g} / \mathrm{l}$, and 50 polymorphs $/ \mathrm{ml}$ with no organisms visible on gram stain. Staphylococcus aureus was cultured. He was treated with cefotaxime and gentamicin and improved becoming fully conscious. A week later he continued to spike nocturnal temperatures. On further questioning he gave a history of a fight with another patient 3 weeks before admission since when he had had slight back pain. A lumbar spine radiograph showed destruction of the L5/S1 disc space and an isotope bone scan showed increased uptake in this region. Antibiotics were changed to flucloxacillin and fusidic acid which were continued for 4 weeks. He made a complete recovery.

Correspondence: H.S. Markus, M.R.C.P.

Accepted: 25 April 1989
Case 2

A 46 year old woman was admitted with a 2 week history of polyuria, polydipsia, blurred vision and confusion. She had lost 2 stone in weight over 2 months. She had experienced lower back pain for 5 weeks. She was pyrexial at $37.6^{\circ} \mathrm{C}$. Blood glucose was $21.5 \mathrm{mmol} / \mathrm{l}$ with normal electrolytes. The following day, despite her glucose being controlled, she remained confused and developed neck stiffness. Lumbar puncture revealed turbid cerebrospinal fluid (CSF) with protein $3 \mathrm{~g} / 1$ and $4 \times 10^{9}$ polymorphs/l. Gram stain showed Gram-positive cocci. She was treated with intravenous benzylpenicillin, flucloxacillin, ampicillin and fucidin. The following day blood and CSF cultures grew Staphyloccus aureus sensitive to penicillin and fusidic acid. Bone scan of the lower thoracic and lumbo-sacral spine showed no increased uptake. Penicillin and fusidic acid were continued and she improved but remained mildly confused and intermittently pyrexial. Five days after admission she had a cardiac arrest and died suddenly. Autopsy revealed slightly oedematous brain with a small amount of meningeal exudate. There was an abscess of the left psoas muscle extending from L4 down over the pelvic brim. This appeared to have originated from osteomyelitis of the L4/5 vertebrae. Pus from the abscess grew Staphylococcus aureus.

\section{Discussion}

Staphylococcus aureus meningitis is rare, accounting for $1-9 \%$ of all bacterial meningitis. ${ }^{1}$ It has a high mortality of $14-77 \%$, although this may reflect the incidence of underlying disease. ${ }^{2}$ In a recent series of 38 cases, 23 were associated with neurosurgical problems including operations, craniospinal defects, trauma, and indwelling ventricular shunts. ${ }^{1}$ The remainder 
were classified as 'spontaneous'; of these 12 had underlying disease, either systemic diseases including renal failure, cirrhosis, and leukaemia, or an underlying staphyloccocal focus of infection, most commonly endocarditis but also cellulitis, abscesses and infected haemodialysis shunts. This 'spontaneous' group had a mortality of $66 \%$.

Vertebral osteomyelitis is a rare but important diagnosis in view of the importance of treatment. The most common neurological complication is cord compression secondary to an epidural abscess, occurring in $3-19 \%$ of cases. ${ }^{3-5}$ Meningitis is extremely rare. Kotrilik and Pechacek reported a fatal case of staphyloccal meningitis in a man with $\mathrm{L} 2 / 3$ osteomyelitis. ${ }^{6}$ Spotkov et al. more recently reported the first case of $S$. aureus meningitis complicating a psoas abscess secondary to $\mathrm{Ll}$ osteomyelitis, similar to our case 2.' A similar case has recently been reported with pneumococcus. ${ }^{8}$ Spread may be either haematogenous or via perforation of the dura.

Vertebral osteomyelitis underlying meningitis may

\section{References}

1. Fong, I.W. \& Ranalli, P. Staphylococcus aureus meningitis. $Q J$ Med 1984, 210: 289-299.

2. Gordon, J.J., Harter, D.H. \& Phair, J.P. Meningitis due to Staphylococcus aureus. Am J Med 1985, 78: 965-969.

3. Ross, D.M. \& Fleming, J.L. Vertebral body osteomyelitis. Clin Orthop 1976, 1976, 118: 190-198.

4. Silverthorn, K.G. \& Gillespie, W.J. Pyogenic spinal osteomyelitis: a review of 61 cases. NZ Med J 1986, 99: $62-65$.

5. Digby, J.M. \& Kersley, J.B. Pyogenic non-tuberculous spinal infection. J Bone Joint Surg (Br) 1979, 61B: 47-55. present a difficult diagnosis as illustrated in our cases. A history of lower back pain may be present, although it may be difficult to elicit in ill or uncooperative patients, as in case 1 . Spinal radiographs may show destructive changes; however, these may be absent early in the disease. An isotope bone scan is usually positive but recently false negative scans have been reported, as in case $2 . .^{9}$ Computed tomography and magnetic resonance imaging may be useful especially in delineating associated abscesses. It is an important diagnosis to make in view of the high mortality of vertebral osteomyelitis, the prolonged antibiotic treatment indicated and the need for bone-penetrating antibiotics. In addition the presence of associated abscesses may require surgical intervention.

\section{Acknowledgement}

We thank Professor J. Hampton for permission to publish case 1 .

6. Kotrilik, J. \& Pechacek, M. Stafylokokova meningitida z osteomyelitidy patere. Cas Lek Ces 1966, 105: 720-722.

7. Spotkov, J., Garber, S. \& Ruskin, J. Staphylococcal meningitis: a complication of psoas abscess. Neurology 1985, 35: 110-111.

8. Francis, N.S., Das, S. \& Tyrrell, P. Osteomyelitis with a rapidly fatal course. $J R$ Soc Med 1988, 81: 51-52.

9. Schlaeffer, F., Mikolich, D.J. \& Mates, S.M. Technetium Tc $99 \mathrm{~m}$ diphosphonate bone scan false normal findings in elderly patients with haematogenous vertebral osteomyelitis. Arch Intern Med 1987, 147: 2024-2026. 